\title{
Ocorrência de uma nova bentonita brasileira nos basaltos intemperizados da formação Mosquito, bacia do Parnaíba, sul do Maranhão
}

\section{(Occurrence of a new brazilian bentonite in the weathered basalts of the Mosquito formation, Parnaiba basin, southern Maranhão, Brazil)}

\author{
S. P. A. Paz , R. S. Angélica ${ }^{1}$, R. F. Neves ${ }^{1,2}$, R. Neumann ${ }^{3}$, G. M. da Costa ${ }^{4}$ \\ ${ }^{1}$ Instituto de Geociências, Laboratório de Caracterização Mineral, \\ ${ }^{2}$ Faculdade de Engenharia Química, Universidade Federal do Pará, Rua Augusto Corrêa s/n, Belém, PA 66075-110 \\ ${ }^{3}$ Centro de Tecnologia Mineral (CETEM) \\ ${ }^{4}$ Departamento de Química, Universidade Federal de Ouro Preto \\ paz@ufpa.br,angelica@ufpa.br
}

\begin{abstract}
Resumo
No município de Formosa da Serra Negra, sul do Maranhão, às margens da rodovia MA006, encontram-se afloramentos de basaltos intemperizados da Formação Mosquito, apresentando textura bastante argilosa e potencial de ser um material bentonítico. Neste trabalho, esse material que vem sendo considerado uma nova ocorrência de bentonita no Brasil e, batizado de Formosa, foi caracterizado por difração de raios X, fluorescência de raios X, microscopia eletrônica de varredura, espectroscopia Mössbauer e capacidade de troca catiônica, em amostra total e após separação granulométrica, e ainda, comparada com duas das principais bentonitas brasileiras, Chocolate e Bofe. Apesar das variações químicas e mineralógicas encontradas, muito comuns nesse tipo de argila, as semelhanças observadas convencem o promissor uso da argila Formosa como bentonita, uma vez que o ponto principal da caracterização está na presença dominante de montmorillonita e baixa concentração de outras fases minerais, mostrando que esse material apresenta mineralogia importante do ponto de vista tecnológico.
\end{abstract}

Palavras-chave: bentonita, argila, esmectita, montmorillonita.

Abstract

Extensive weathered basalt outcrops can be found in the vicinities of the Formosa da Serra Negra town, southern of Maranhão state, northern Brazil. They exhibit typical clay texture and an enormous potential to be used and explored as bentonite. This work deals with the chemical and mineralogical characterization of this material that has been named as Formosa Bentonite. The main following methods and analytical techniques were used: XRD, XRF, SEM, Mössbauer spectroscopy and CEC in total sample and after grain size separation. Two samples from the main Brazilian "Paraiba bentonites" (Chocolate and Bofe types) were used for comparison purposes. The results show that the Formosa bentonite exhibit some chemical and mineralogical differences in comparison with the two reference samples, although the main and most important feature is the presence of montmorillonite as the major mineral phase.

Keywords: bentonite, clay, esmectite, montmorillonite.

\section{INTRODUÇÃO}

A bacia sedimentar do Parnaíba, também conhecida por bacia do Meio-Norte ou bacia do Maranhão, localiza-se na região nordeste ocidental do território brasileiro, ocupando uma área de aproximadamente $600.000 \mathrm{~km}^{2}$, compreendendo parte dos estados do Maranhão, Piauí, Tocantins e, restritamente, do Ceará [1]. Sabe-se que a participação da mineração na economia dessa região é ainda muito pequena, praticada quase sempre por processos rudimentares ou semi-industriais, que se limitam à extração de poucos bens minerais, especialmente não-metálicos. Dentre esses, algumas argilas industriais importantes já são conhecidas, associadas a rochas sedimentares, como os depósitos de palygorskita de Guadalupe, Formações Poti e Piauí, sudoeste do Piauí, utilizada como descorante de óleos vegetais, e lavrados desde os anos 70 [2]. Outras ocorrências como as de esmectitas das Formações Motuca e Pedra de Fogo apresentam potencial para jazimentos, porém sem notícia de explotação [3, 4]. Além dessas ocorrências sedimentares já conhecidas, importantes depósitos esmectíticos residuais podem estar associados ao intemperismo de rochas máficas (basaltos) da Formação Mosquito, que ocorrem na forma de derrames, observados em vários trechos da rodovia Belém-Brasília (BR-010), próximo a divisa entre os estados do Maranhão e Tocantins e em vários trechos das rodovias estaduais que cortam, principalmente, o 
sul do Maranhão $[3,5,6]$.

As argilas esmectíticas são materiais constituídos por um ou mais argilominerais do grupo das esmectitas, sendo a montmorillonita a espécie principal constituinte das bentonitas (insumo de grande valor comercial) [7, 8]. Minerais acessórios como quartzo, cristobalita, micas e feldspatos, além de outros argilominerais como os do grupo da caulinita podem estar associados. A esmectita é um silicato de alumínio hidratado, cuja estrutura cristalina a classifica como filossilicato (silicato de estrutura em folhas), em grego, phyllos significa "folha" ou lamela. É formada pelo empilhamento regular de camadas 2:1, isto é, cada camada é constituída por um "sanduíche" de duas folhas de tetraedros $\mathrm{SiO}_{4}$ contendo uma folha de octaedros $\mathrm{Al}(\mathrm{OH})_{3}$ ou $\mathrm{Mg}(\mathrm{OH})_{2}$ como "recheio". Permite, durante seu processo de formação, significantes substituições nos sítios tetraédricos, quase sempre $\mathrm{Si}^{4+}$ por $\mathrm{Al}^{3+}$, ocasionalmente $\mathrm{Fe}^{3+}$ ou $\mathrm{Fe}^{2+}$ e, nos octaédricos, em geral $\mathrm{Al}^{3+}$ por $\mathrm{Mg}^{2+}, \mathrm{Fe}^{2+}$ e $\mathrm{Fe}^{3+}$, ocasionalmente $\mathrm{Mn}^{2+}, \mathrm{Zn}^{2+}, \mathrm{Li}^{+}$, entre muitos outros, o que gera excesso de carga negativa, precisando de cargas compensadoras (cátions) para balancear [7-11]. Em geral, esses cátions são $\mathrm{Ca}^{2+}, \mathrm{Mg}^{2+}, \mathrm{Na}^{+} \mathrm{e} \mathrm{K}$, que ocupam os espaços interlamelares e são facilmente trocados. A alta capacidade de troca desses cátions por outros externos, em solução, por exemplo, tem justificado seu alto emprego.

Geologicamente, bentonita é um termo para a rocha constituída dominantemente por esmectita, montmorillonita sódica, formada pela desvitrificação e subseqüente alteração química de um material vítreo, usualmente tufos ou cinzas vulcânicas, de preferência félsicas, com capacidade de adsorver grande quantidade de água, formando uma massa volumosa semelhante a uma gelatina [12]. Essa denominação foi utilizada pela primeira vez em 1898, para esse tipo de montmorillonita sódica que ocorria próximo ao Forte Benton, na região Rock Creed, estado de Wyoming, EUA [12]. No entanto, por falta de produtos naturais iguais ao norte-americano, outros países passaram a extrair esmectitas montmorilloníticas diversas, cujas propriedades se assemelham àquelas, e que depois de tratadas com sódio, eram comercializadas também com o nome de bentonita, o que generalizou esse termo [12]. Tecnologicamente, passouse aceitar como bentonita, qualquer argila esmectítica que apresente propriedades análogas às das bentonitas tradicionais e/ou se já forem utilizadas comercialmente como tal, independente de sua gênese [13-17]. Dessa maneira, passaram a existir dois tipos de argilas bentoníticas para uso industrial: a) as que incham em presença de água, nas quais o $\mathrm{Na}^{+}$é o cátion interlamelar predominante e b) as que não incham ou incham muito pouco em presença de água, que têm $\mathrm{Ca}^{2+}$ e/ou $\mathrm{Mg}^{2+}$ como cátion interlamelar predominante e que em geral são ativadas com carbonato de sódio, para serem transformadas em sódicas. Esse processo de ativação foi desenvolvido e patenteado na Alemanha, no ano de 1933, pela empresa Erbsloh \& Co. e é atualmente utilizado pelos países que não dispõem de bentonita sódica natural $[15$, 16]. As bentonitas comercializadas no Brasil são sódicas naturais importadas ou $\mathrm{Ca}, \mathrm{Mg}$-bentonitas nacionais ativadas sodicamente mediante tratamento com barrilha [15]. Segundo o Departamento Nacional de Produção Mineral 2008 [17], o Brasil é responsável por $2 \%$ da produção mundial de bentonita, concentrando cerca de $80 \%$ da produção nacional no estado da Paraíba, mais precisamente no município de Boa Vista. Estas argilas bentoníticas são consideradas as melhores do país e desde sua descoberta, início dos anos 60 , são extraído elevados volumes que já levaram boa parte das variedades mineralógicas à exaurição [14]. Oficialmente 14 empresas atuam neste ramo da mineração no país. A maior delas é a Bentonit União Nordeste que produziu, ainda segundo o DNPM 2008, 48,1\% da bentonita bruta. Nesse mesmo ano, os investimentos na mineração de bentonita brasileira alcançaram R $\$ 3.748 .770,00$, dos quais $43,3 \%$ foram para equipamentos, $25,6 \%$ para infra-estrutura, $8,2 \%$ para desenvolvimento de mina, $8,1 \%$ geologia e pesquisa mineral, $7 \%$ saúde e segurança no trabalho, 4,1\% meio ambiente, $1,6 \%$ estudos geotécnicos, $1,2 \%$ inovações tecnológicas, $0,7 \%$ caracterização tecnológica do minério. Com investimentos previstos até 2011 na mineração e beneficiamento da bentonita no Brasil da ordem de R $\$ 9.987 .000,00$ [17].

Os principais usos da bentonita são: como agente tixotrópico de fluidos de perfuração de poços de petróleo e de água; aglomerante de areias de moldagem usadas em fundição; pelotização de minérios de ferro; descoramento de óleos e granulado sanitário para gatos. Mas, a distribuição de seu consumo varia de modo significante de acordo com o país [16]. No Brasil, as principais aplicações da bentonita são na pelotização de minério de ferro e fluidos de perfuração, enquanto nos EUA são no granulado sanitário para gatos e fluidos de perfuração. Nesse contexto, acredita-se que depósitos esmectíticos derivados de basaltos intemperizados, como os da bacia do Parnaíba, podem vir a ser uma alternativa interessante à futura escassez das argilas bentoníticas da Paraíba, assim como, somar a produção nacional existente para suprir a demanda interna, que só tende a aumentar dado ao elevado crescimento industrial do país. Para isso, são necessários estudos de detalhe dessas ocorrências, uma vez que, para as aplicações industriais, uma identificação completa do tipo de argilomineral e de suas propriedades é importante e indispensável no estabelecimento de seu melhor uso. Assim, o objetivo principal desse trabalho é a caracterização química e mineralógica desse material, aqui considerado como uma nova ocorrência de bentonita no Brasil.

\section{MATERIAIS E MÉTODOS}

A argila investigada foi coletada em um afloramento de basalto intemperizado da Formação Mosquito, (coordenadas

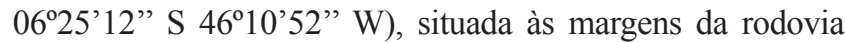
(MA 006), município de Formosa da Serra Negra, sul do Maranhão. Trata-se de saprólito de basalto amigdaloidal, com mais de dois metros de espessura, intercalado com níveis de arenitos associados a esta unidade ou relacionados à Formação Corda. Por apresentar mineralogia dominada por esmectitamontmorillonita, e grande extensão em área de ocorrência, esse material foi denominado, informalmente, de Bentonita Formosa, nome que faz menção ao seu lugar de ocorrência e 
a uma esmectita com potencial de aproveitamento econômico. As bentonitas comerciais nacionais Chocolate e Bofe, provenientes do município de Boa Vista (PB), adquiridas na forma in natura de uma mineradora que as exploram, foram utilizadas como referências no estudo de caracterização química e mineralógica da bentonita Formosa. Os nomes das amostras, a origem, cor e os códigos adotados neste trabalho estão indicados na Tabela I.

Tabela I - Descrição das amostras.

[Table I - Description of the samples.]

\begin{tabular}{llll}
\hline Nome da amostra & Origem & Código & Cor \\
\hline Bentonita Formosa & Maranhão & BF & $\begin{array}{l}\text { Marrom } \\
\text { avermelhado }\end{array}$ \\
$\begin{array}{llll}\text { Bentonita } \\
\text { Chocolate }\end{array}$ & Paraíba & BC & Marrom \\
Bentonita Bofe & Paraíba & BB & Bege \\
\hline
\end{tabular}

Cerca de $4 \mathrm{~kg}$ de bentonita Formosa composta de material coletado em diferentes pontos do depósito descrito anteriormente foi britada em britador de mandíbulas Retsch BB2. Após moagem em moinho orbital (Shatter Box) Hgherzog HSM100, a amostra foi classificada abaixo de $0,15 \mathrm{~mm}$ (ASTM 100) em peneiramento manual. O material retido nesta peneira foi novamente submetido ao moinho até que passasse a totalidade da amostra. O quarteamento foi realizado segundo método de pilha alongada, garantindo homogeneização e com isso uma amostra representativa. As amostras de referência $\mathrm{BC}$ e BB foram moídas, classificadas e quarteadas segundo procedimento semelhante ao da amostra $\mathrm{BF}$. O fracionamento granulométrico da bentonita Formosa foi feito a partir de 500 $\mathrm{g}$ da amostra, que foi dispersa em $1 \mathrm{~L}$ de água deionizada em banho de ultra-som por $30 \mathrm{~min}$. Após a dispersão, realizou-se o fracionamento em cinco faixas granulométricas: grossa1 $(\varnothing$ $>75 \mu \mathrm{m})$, grossa2 $(\varnothing>20 \mu \mathrm{m})$, intermediária $(5<\varnothing<20$ $\mu \mathrm{m})$, final $(\varnothing<5 \mu \mathrm{m})$ e fina $2(\varnothing<2 \mu \mathrm{m})$. Com o emprego de classificação em peneiramento a úmido obteve-se as frações grossa1 e grossa2; através da sedimentação gravitacional obteve-se: a fração fina1, sobrenadante retirado após $24 \mathrm{~h}$, a fração fina2, sobrenadante retirado após 96 h, e a fração intermediária, produto de sedimentação. Essas amostras receberam as seguintes identificações: BFG1 (grossa1), BFG2 (grossa2), BFI (intermediária), BFF1 (fina1) e BFF2 (fina2). Estes procedimentos e as análises realizadas em cada amostra gerada estão apresentados no fluxograma da Fig. 1. Além dessas frações, obteve-se ainda uma pequena quantidade de fração magnética (BFMAG) separada por Tubo Davis.

Todas as amostras foram secas a $105{ }^{\circ} \mathrm{C}$ e submetidas à caracterização por diversas técnicas experimentais.

Fluorescência de raios $X(F R X)$ : o espectrômetro utilizado foi PANalytical Axios-Minerals, com tubo de raios $\mathrm{X}$ de ródio e amostra de pastilha fundida ( $1 \mathrm{~g}$ de amostra para $8 \mathrm{~g}$ de metaborato de lítio), com o programa IQ+, PANalytical.

Difração de raios $X(D R X)$ : as análises foram realizadas em difratômetro PANalytical X'Pert Pro $\operatorname{MPD}(\theta / \theta)$, radiação $\mathrm{Cuk} \alpha 1=1,540598 \AA$, filtro $\mathrm{k} \beta$ de Ni. As condições

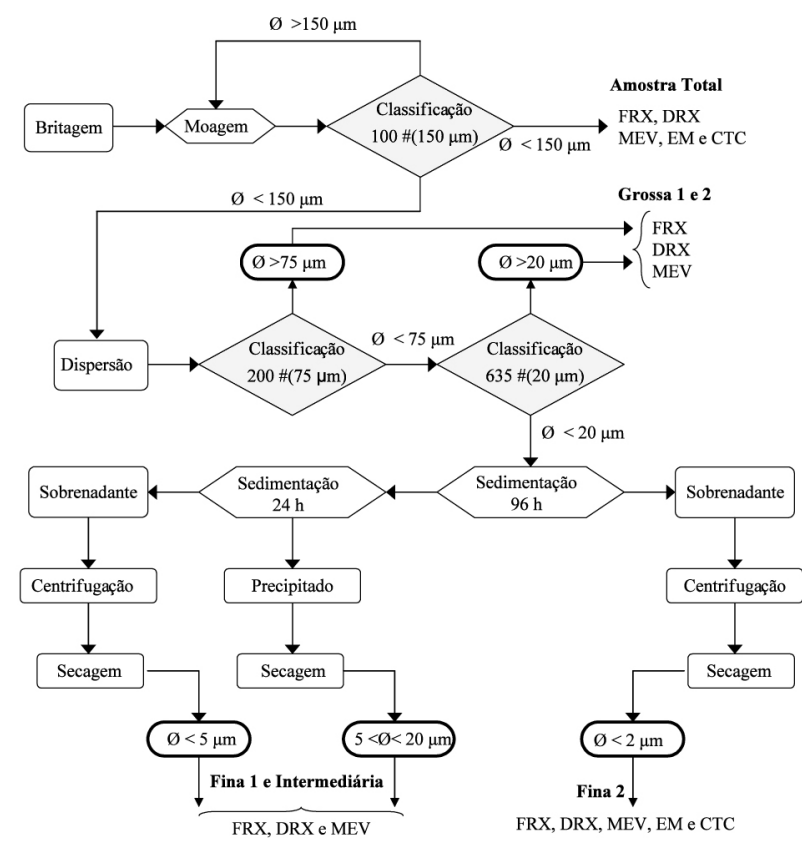

Figura 1: Fluxograma do fracionamento granulométrico.

[Figure 1: Flowchart of the grain size separation.]

instrumentais foram: - método do pó total: varredura de 2 a $52^{\circ}$ em $2 \theta, 40 \mathrm{kV}, 30 \mathrm{~mA}$, passo $0,02^{\circ} \mathrm{em} 2 \theta$ e tempo/passo $60 \mathrm{~s}$, fenda divergente de $1 / 8^{\circ}$ e anti-espalhamento de $1 / 4^{\circ}$, máscara de $10 \mathrm{~mm}$, amostra em movimentação circular 1 rotação/s; método de orientação em lâmina de vidro: varredura de 3 a $35^{\circ}$ em $2 \theta ; 40 \mathrm{kV}, 30 \mathrm{~mA}$, passo $0,02^{\circ}$ em $2 \theta$ e tempo/passo $10 \mathrm{~s}$, fenda divergente de $1 / 8^{\circ}$ e anti-espalhamento de $1 / 4^{\circ}$, máscara de $10 \mathrm{~mm}$, sem rotação. A mesma amostra foi analisada três vezes, a primeira em sua forma natural, a segunda saturada com etilenoglicol e terceira calcinada a $550^{\circ} \mathrm{C}$ por $2 \mathrm{~h}$.

Microscopia eletrônica de varredura (MEV): as micrografias foram obtidas em um microscópio LEO 1430, nas condições de análises para as imagens de elétrons secundários, corrente $90 \mu \mathrm{A}$, voltagem $20 \mathrm{kV}$, distância de trabalho $10 \mathrm{~mm}$.

Espectroscopia Mössbauer (EM): os espectros foram coletados à temperatura ambiente em um espectrômetro operando no modo de aceleração constante e um sinal de referência triangular. A acumulação dos dados foi feita com um analisador multicanal com 512 canais e intervalo de velocidade $-10 \mathrm{a}+10 \mathrm{~mm} / \mathrm{s}$.

Capacidade de troca cationica (CTC): foi usado o método do azul de metileno [18] para determinar as CTCs das bentonitas BF, BC e BB (amostra total) e o método do acetato de amônio [19] para determinar as CTCs parciais e total da fração argila da bentonita Formosa (BFF2).

\section{RESULTADOS E DISCUSSÃO}

\section{Amostra Total - Amostras BF, BC e BB}

A composição química da bentonita Formosa (BF), assim como das amostras de referência (BC e BB), está apresentada na Tabela II. Nota-se que $\mathrm{SiO}_{2}$ e $\mathrm{Al}_{2} \mathrm{O}_{3}$ são os componentes 
Tabela II - Composição química (\% massa) das bentonitas BF, BC e BB (amostra total).

[Table II - Chemical composition (wt.\%) of the BF, BC and BB bentonites (bulk sample).]

\begin{tabular}{ccccccccccccc}
\hline Amostra & $\mathrm{SiO}_{2}$ & $\mathrm{Al}_{2} \mathrm{O}_{3}$ & $\mathrm{Fe}_{2} \mathrm{O}_{3}$ & $\mathrm{MgO}$ & $\mathrm{CaO}$ & $\mathrm{K}_{2} \mathrm{O}$ & $\mathrm{Na}_{2} \mathrm{O}$ & $\mathrm{MnO}$ & $\mathrm{TiO}_{2}$ & $\mathrm{P}_{2} \mathrm{O}_{5}$ & P.F. & Total \\
\hline $\mathrm{BF}$ & 48,13 & 17,40 & 14,08 & 3,63 & 0,27 & 1,59 & - & 0,17 & 1,49 & 0,05 & 13,08 & 99,89 \\
$\mathrm{BC}$ & 56,76 & 15,37 & 10,19 & 2,76 & 0,53 & 0,29 & 0,86 & - & 0,70 & 0,19 & 12,12 & 99,80 \\
$\mathrm{BB}$ & 64,07 & 14,63 & 6,87 & 1,91 & 0,44 & 0,19 & 0,61 & - & 0,70 & 0,03 & 10,53 & 100,00 \\
\hline
\end{tabular}

$-=$ abaixo do limite de detecção e P.F. = Perda ao fogo determinada com a queima a $1000{ }^{\circ} \mathrm{C}$, após secagem a $105^{\circ} \mathrm{C}$.

principais em todas elas, sendo que $\mathrm{BF}$ apresenta o maior teor de $\mathrm{Al}_{2} \mathrm{O}_{3}$, embora apresente o menor teor de $\mathrm{SiO}_{2}$. A $\mathrm{BF}$ possui alto teor de $\mathrm{Fe}_{2} \mathrm{O}_{3}$, uma característica das argilas esmectíticas brasileiras. E mais, apresenta teores mais baixos de $\mathrm{CaO}$ e $\mathrm{Na}_{2} \mathrm{O}$ e maiores de $\mathrm{MgO}, \mathrm{K}_{2} \mathrm{O}, \mathrm{TiO}_{2}$ e $\mathrm{MnO}$, em relação às amostras $\mathrm{BC}$ e $\mathrm{BB}$. Os valores de perda ao fogo apresentados pelas bentonitas analisadas estão próximos.

De modo geral, as composições químicas das bentonitas costumam variar, devido às inúmeras substituições isomórficas nas esmectitas e, por conta das impurezas associadas (minerais acessórios presentes). Sendo assim, uma melhor interpretação química pode ser feita em conjunto com os resultados mineralógicos, descritos a seguir.

Foi identificada por difratometria de raios $\mathrm{X}$ na amostra BF (amostra total), Fig. 2, presença predominante de esmectita-montmorillonita (15,3, 4,47 e 1,49 $\AA)$ e traços de caulinita (7,2, 2,56 e 2,34 $\AA$ ), K-feldspato (4,20, 3,26 e 3,23 $\AA$ ) e hematita $(2,70,2,51$ e $1,84 \AA$ ). Com a análise da fração argila (BFF2) no modo desorientado, de 60 a $64^{\circ}$ em $2 \theta$, identificou-se a posição $1,495 \AA$ do plano 060 , impressão digital da montmorillonita. Os difratogramas da amostra

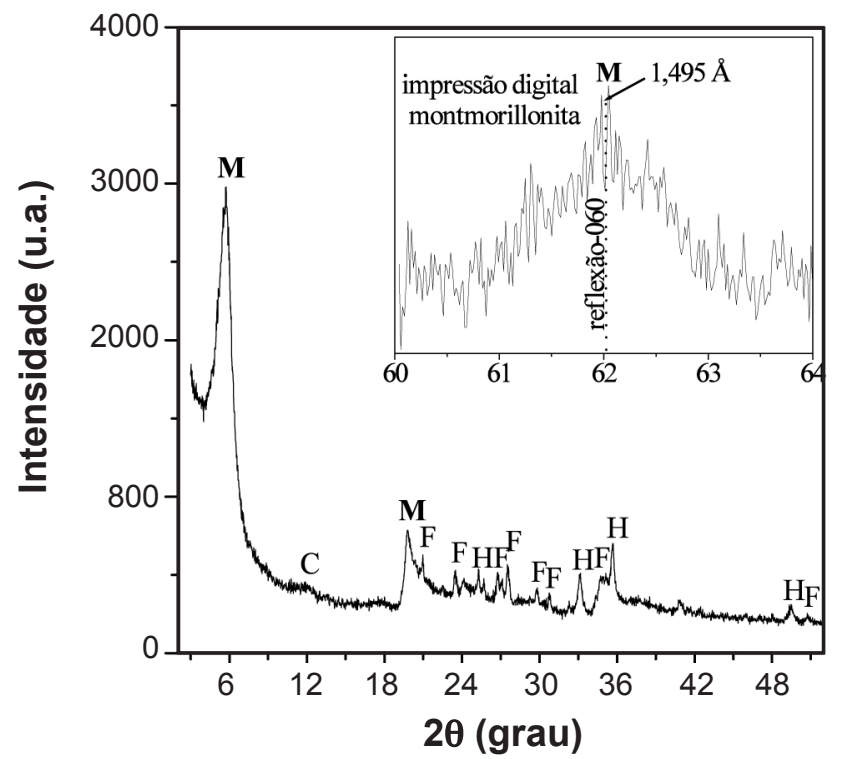

Figura 2: Difratogramas de raios $\mathrm{X}$ de $\mathrm{BF}$ amostra total e fração argila (BFF2) desorientada na região impressão digital da esmectita, ( $\mathrm{M}=$ montmorillonita, $\mathrm{C}=$ caulinita, $\mathrm{F}=\mathrm{K}$-feldspato, $\mathrm{H}=$ hematita $)$. [Figure 2: X-ray diffraction patterns of BF bulk sample and unoriented clay fraction (BFF2) in the "fingerprint" region of the esmectite, $(M=$ montmorillonite, $C=$ Kaolinite, $F=K$-feldspar, $H=$ hematite).]

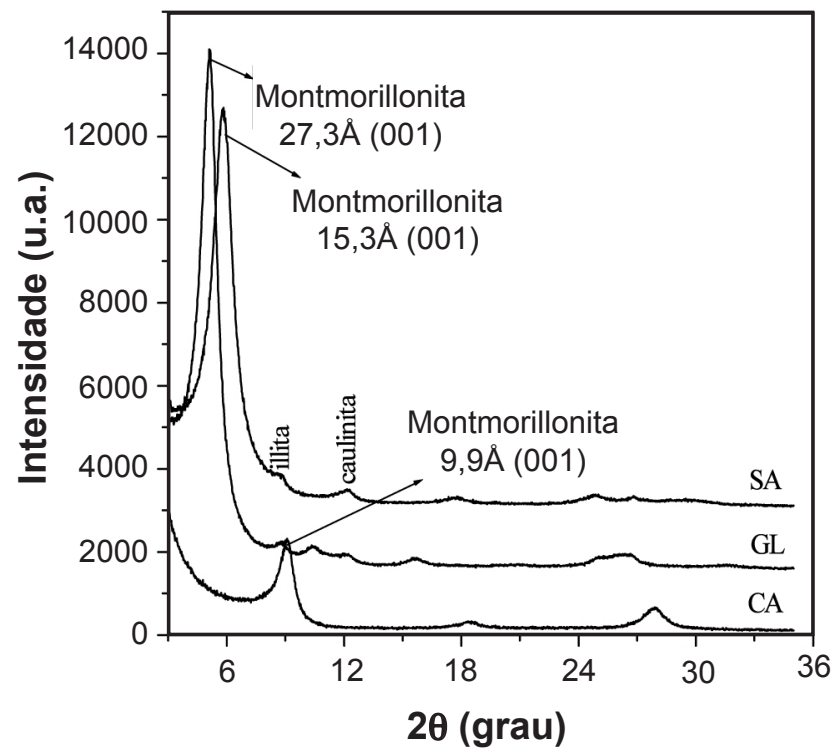

Figura 3: Difratogramas da fração argila (BFF2) orientada: seca ao ar (SA), glicolada (GL) e calcinada a $550{ }^{\circ} \mathrm{C}(\mathrm{CA})$.

[Figure 3: $X$-ray diffraction patterns of the oriented clay fraction (BFF2): air-dried (SA), glycolated (GL) and heated at $550^{\circ} \mathrm{C}(\mathrm{CA})$.]

BFF2 no modo orientada natural, saturada com etilenoglicol e calcinada (Fig. 3), confirmaram a presença predominante da montmorillonita, pela característica expansiva, $\mathrm{d}_{001}$ indo de 15,3 para 17,3 Å quando glicolada, e colapsando para 9,9 $\AA$ quando calcinada. Foi possível identificar ainda, na fração BFF2, traços de illita (10 e $5 \AA$ ).

Observam-se semelhanças entre os difratogramas das amostras BF, BC e BB (Fig. 4), principalmente com respeito aos planos principais da esmectita, (001) e (100). A caulinita aparece nas amostras BF e BB, enquanto o quartzo está presente nas amostras BB e BC. Outras impurezas como K-feldspato e hematita somente foram identificadas na amostra BF; cristobalita na amostra BB e goethita na amostra BC.

A partir dos resultados da difração de raios X (Figs. 2, 3 e 4) e da composição química (Tabela II), pode-se fazer as seguintes correlações: - A amostra BF apresenta o menor teor de $\mathrm{SiO}_{2}$, quando comparada com as amostras de referência, porém não apresenta quartzo e/ou cristobalita, o que pode justificar os maiores teores em $\mathrm{BC}$ e $\mathrm{BB}$; - a amostra $\mathrm{BF}$ apresenta teor de $\mathrm{Al}_{2} \mathrm{O}_{3}$ maior que $\mathrm{BC}$ e $\mathrm{BB}$, o que leva a supor menores substituições isomórficas nos sítios octaédricos e/ou maiores substituições isomórficas de $\mathrm{Al}$ por Si nos sítios tetraédricos. Talvez por isso menor teor 


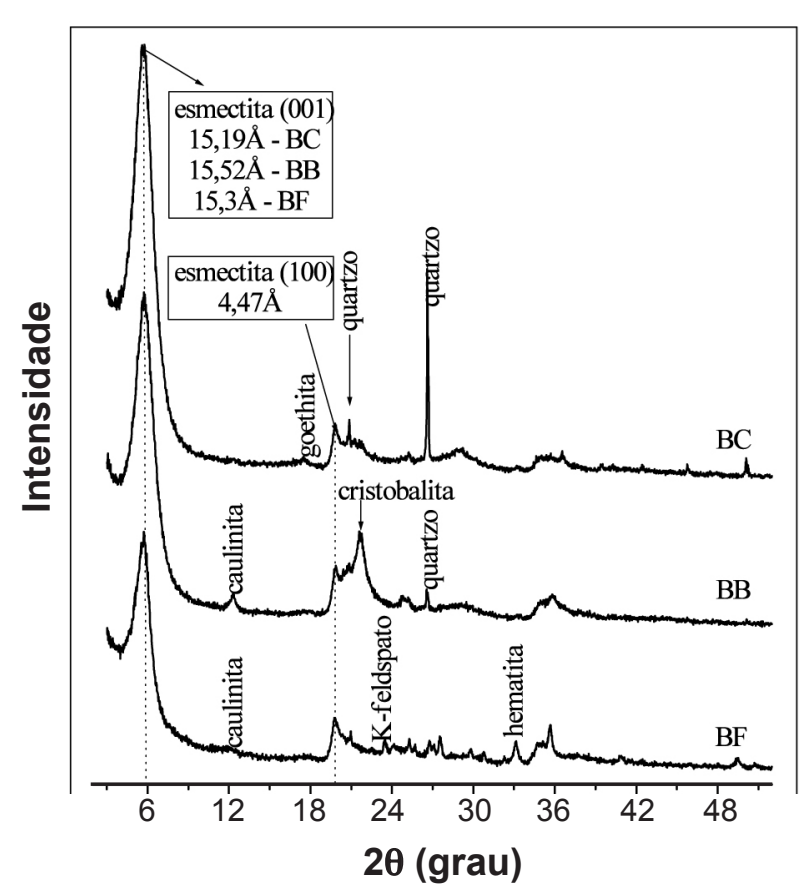

Figura 4: Difratogramas de raios $\mathrm{X}$ das amostras BF, BC e BB (amostra total).

[Figure 4: X-ray diffraction patterns of $B F, B C$ and $B B$ (bulk sample).]

de $\mathrm{SiO}_{2}$, já que o feldspato colabora igualmente com $\mathrm{Si}$ e $\mathrm{Al}$, assim como a caulinita, que também está presente em BB, nem por isso aumenta seu valor de $\mathrm{Al}_{2} \mathrm{O}_{3}$; - os valores de $\mathrm{d}_{001}$ de 14,89 a $15,5 \AA$ indicam a presença de $\mathrm{Ca}-\mathrm{Mg}$-esmectita $[13,20]$. É o caso das amostras estudadas (BF, BC e BB), que apresentam $\mathrm{d}_{001}$ nessa faixa e quantidades de $\mathrm{Ca}$ e/ou $\mathrm{Mg}$ compatíveis; - o teor relativamente elevado de $\mathrm{K}_{2} \mathrm{O}$ na $\mathrm{BF}$ deve-se a presença de illita e, principalmente, $\mathrm{K}$-feldspato; - o teor de $\mathrm{TiO}_{2}$ na amostra $\mathrm{BF}$ esta relacionado a presença de anatásio e possivelmente de uma titanomagnetita, somente identificados na fração magnética (vide a seguir).

Os valores de CTC das amostras BF, BC e BB (amostra total) encontram-se na Tabela III. Sabe-se que, por algumas limitações do método do azul de metileno (AM), os valores encontrados são quase sempre inferiores aos obtidos por outros métodos, como por exemplo, acetato de amônio ou cloreto de bário, que são mais trabalhosos e demorados, ao contrário do $\mathrm{AM}$, que é simples e rápido. Todavia, o resultado mostra uma tendência, possibilitando uma comparação entre amostras submetidas às mesmas condições. Assim, obteve-se que $\mathrm{BC}$ apresenta maior $\mathrm{CTC}$ que $\mathrm{BB}$ e BF, condizente com a mesma ordem de grandeza apresentada por outros trabalhos $[21,22]$. E ainda, que o resultado obtido para as amostras BB e BF apresenta, aproximadamente, o mesmo valor de CTC.

$\mathrm{Na}$ Tabela IV estão discriminadas as CTCs parciais e total da fração argila da bentonita Formosa (BFF2), obtidas com o método do acetato de amônio. Observa-se que o $\mathrm{Mg}^{2+}$ é o cátion trocável majoritário, em $\mathrm{pH} \sim 7$, mesmo com seletividade do $\mathrm{Ca}^{2+}>\mathrm{Mg}^{2+}$ [23]. Isso implica em uma montmorillonita magnesiana, que é de certa forma menos comum, comparada com as bentonitas comercialmente
Tabela III - Capacidade de Troca Catiônica determinada pelo método azul de metileno.

[Table III - Cation Exchange Capacity determined by method of methylene blue adsorption.]

\begin{tabular}{ccc}
\hline Amostra & $\begin{array}{c}\text { CTC } \\
\text { (meq/100 g de argila) } \\
\text { (este trabalho) }\end{array}$ & $\begin{array}{c}\text { CTC } \\
\text { (meq/100 g de argila) } \\
\text { (literatura) }\end{array}$ \\
\hline $\mathrm{BF}$ & 50,5 & - \\
$\mathrm{BC}$ & 68,1 & $106,0[21] ; 92,0[22]$ \\
$\mathrm{BB}$ & 49,2 & $74,0[21] ; 76,0[22]$ \\
\hline
\end{tabular}

Tabela IV - Capacidade de Troca Catiônica de BFF2 parcial e total determinada pelo método acetato de amônio.

[Table IV - Cation Exchange Capacity of BFF2 partial and total determined by method ammonium acetate.]

\begin{tabular}{ccccc}
\hline \multicolumn{5}{c}{$\mathrm{meq} / 100 \mathrm{~g}$ de argila } \\
\hline $\mathrm{Mg}^{2+}$ & $\mathrm{Ca}^{2+}$ & $\mathrm{K}^{+}$ & $\mathrm{Na}^{+}$ & $\mathrm{CTC}^{*}$ \\
60,19 & 9,88 & 1,41 & 3,35 & 74,83 \\
\hline
\end{tabular}

* calculada pela soma $\mathrm{Mg}+\mathrm{Ca}+\mathrm{K}+\mathrm{Na}$

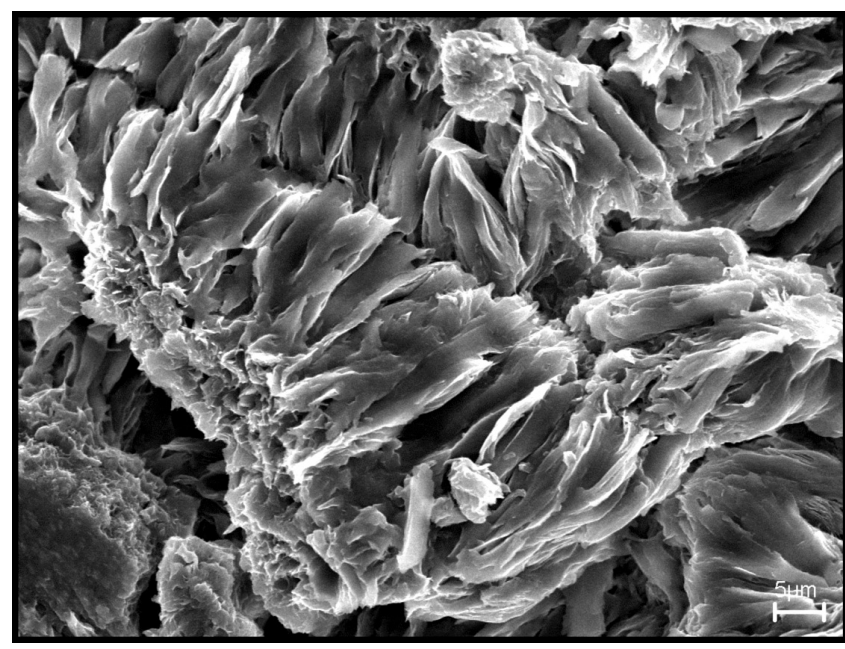

Figura 5: Micrografia obtida por microscopia eletrônica de varredura de BF (amostra total).

[Figure 5: SEM micrograph of BF (bulk sample).]

conhecidas, que em geral são cálcicas ou sódicas, sendo as cálcicas as mais comuns, como é o caso das brasileiras BC e BB $[14,15]$. Além disso, vale ressaltar que, mesmo em trabalhos científicos sobre esmectitas, muitos não fazem uma caracterização mineralógica e química tão minuciosa, limitando-se a caracterizar, por difração de raios X, apenas a espécie dominante, em geral como montmorillonita.

A micrografia (MEV) da amostra global BF é apresentada na Fig. 5. Observa-se a morfologia típica de esmectita: grandes ondulações que indicam o empilhamento das folhas muito extensas [11] ou morfologia tipo rosa ou roseta [24].

\section{Frações Granulométricas - Amostra BF}

Os resultados da composição química das frações 
Tabela V - Composição química (\% massa) das frações granulométricas da amostra BF.

[Table V-Chemical composition (wt.\%) of the grain size fractions of BF.]

\begin{tabular}{lllllllllllll}
\hline \multicolumn{1}{c}{ Amostra } & $\mathrm{SiO}_{2}$ & $\mathrm{Al}_{2} \mathrm{O}_{3}$ & $\mathrm{Fe}_{2} \mathrm{O}_{3}$ & $\mathrm{MgO}$ & $\mathrm{CaO}$ & $\mathrm{K}_{2} \mathrm{O}$ & $\mathrm{Na}_{2} \mathrm{O}$ & $\mathrm{MnO}$ & $\mathrm{TiO}_{2}$ & $\mathrm{P}_{2} \mathrm{O}_{5}$ & P.F. & Total \\
\hline $\mathrm{BFG} 1(\varnothing>75 \mathrm{~m})$ & 49,25 & 16,03 & 16,03 & 4,55 & 0,28 & 0,88 & - & 0,19 & 0,84 & 0,03 & 11,92 & 100,00 \\
$\mathrm{BFG}(\varnothing>20 \mu \mathrm{m})$ & 47,68 & 16,57 & 16,07 & 4,24 & 0,30 & 1,28 & - & 0,11 & 1,40 & 0,04 & 12,3 & 100,00 \\
$\mathrm{BFI}(5<\varnothing<20 \mu \mathrm{m})$ & 49,64 & 17,86 & 14,5 & 2,93 & 0,21 & 3,23 & - & 0,19 & 2,11 & 0,03 & 9,28 & 99,98 \\
$\mathrm{BFF} 1(\varnothing<5 \mu \mathrm{m})$ & 50,43 & 21,74 & 9,50 & 3,80 & 0,23 & 1,25 & - & 0,09 & 0,57 & 0,03 & 12,27 & 99,90 \\
$\mathrm{BFF} 2(\varnothing<2 \mu \mathrm{m})$ & 51,65 & 21,58 & 8,51 & 3,87 & 0,29 & 0,90 & - & - & 0,47 & - & 12,7 & 99,98 \\
\hline
\end{tabular}

- = abaixo do limite de detecção e P.F. = Perda ao fogo determinada com a queima a $1000{ }^{\circ} \mathrm{C}$, após secagem a $105^{\circ} \mathrm{C}$.

granulométricas da amostra BF estão apresentados na Tabela V. $\mathrm{SiO}_{2}$ e $\mathrm{Al}_{2} \mathrm{O}_{3}$ são os componentes principais, com razão $\mathrm{SiO}_{2} / \mathrm{Al}_{2} \mathrm{O}_{3}$ acima de 2 em todas elas. As frações grossas (BFG1 e BFG2) apresentam os mais altos teores de $\mathrm{Fe}_{2} \mathrm{O}_{3}$, estando este elemento quase que na mesma concentração do $\mathrm{Al}_{2} \mathrm{O}_{3}$, além dos maiores teores de $\mathrm{MgO}$. A fração intermediaria $(\mathrm{BFI})$, apresenta os maiores teores de $\mathrm{K}_{2} \mathrm{O}$ e $\mathrm{TiO}_{2}$, e menor quantidade de perda ao fogo. E como era de se esperar, as frações finas (BFF1 e BFF2), apresentam os maiores teores de $\mathrm{SiO}_{2}, \mathrm{Al}_{2} \mathrm{O}_{3}$ e perda ao fogo, e menores de $\mathrm{Fe}_{2} \mathrm{O}_{3}, \mathrm{MnO}$ e $\mathrm{TiO}_{2}$, caracterizando as frações com menor quantidades de impurezas e maiores concentração de argilominerais, principalmente montmorillonita.

Os difratogramas de raios $\mathrm{X}$ das cinco frações granulométricas da amostra BF estão apresentados na Fig. 6. Pode-se observar que montmorillonita está presente em todas elas, estando, como esperado, em maior concentração nas frações BFF1 e BFF2. Na Fig. 7 tem-se o difratograma de raios $\mathrm{X}$ da fração magnética (BFMAG), em que se identificou magnetita além de montmorillonita, hematita e anatásio.

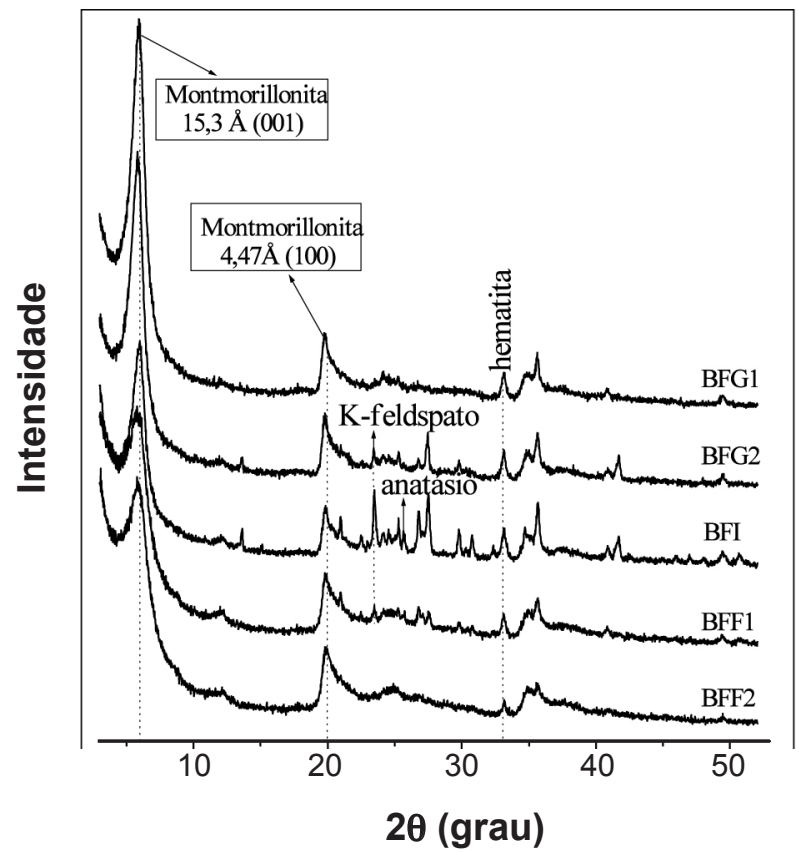

Figura 6: Difratogramas de raios $\mathrm{X}$ das frações granulométricas BFG1, BFG2, BFI, BFF1 e BFF2.

[Figure 6: $X$-ray diffraction patterns of the grain size fractions $B F G 1, B F G 2, B F I, B F F 1$ and $B F F 2$.]

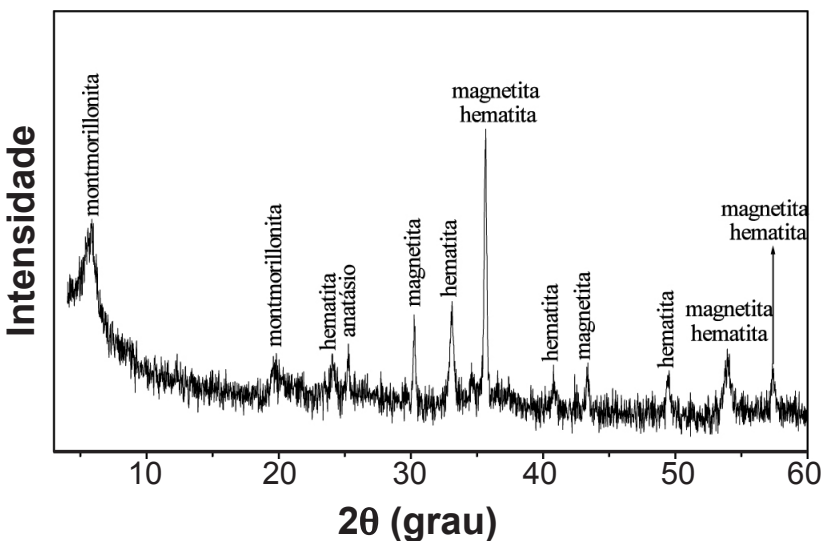

Figura 7: Difratograma de raios X da fração magnética (BFMAG). [Figure 7: X-ray diffraction pattern of the magnetic fraction (BFMAG).]

A partir dos resultados da difração de raios X (Fig. 6 e 7) e da composição química (Tabela V) é possível fazer algumas correlações:- a fração BFI apresentou o maior teor de $\mathrm{K}_{2} \mathrm{O}$ e menor valor de perda ao fogo dentre as cinco frações granulométricas estudadas, isso porque essa fração foi a que mais concentrou K-feldspato; - o baixo teor de $\mathrm{K}_{2} \mathrm{O}$ nas amostras BFG1 e BFF2, condiz exatamente com a ausência de K-feldspato nessas frações. Portanto esses teores devem estar relacionados à montmorillonita e illita; - a percentagem de $\mathrm{CaO}$ que permanece quase constante em todas as frações, refere-se praticamente a montmorillonita; o teor de $\mathrm{TiO}_{2}$ está relacionado ao anatásio e possivelmente a uma titanomagnetita. Minerais que só aparecem nos

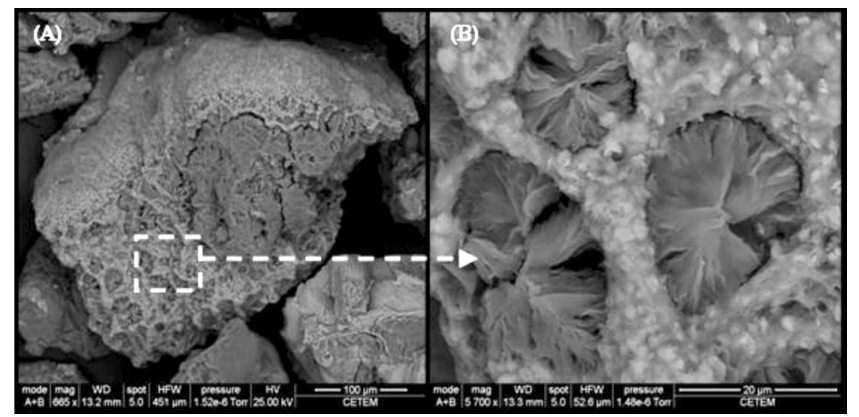

Figura 8: Micrografias obtidas por microscopia eletrônica de varredura da fração BFG1: (A) aglomerado e (B) detalhe da região destacada em A.

[Figure 8: SEM micrographs of the BFG1 fraction: (A) agglomerate and $(B)$ detail of the marked region in $A$.] 


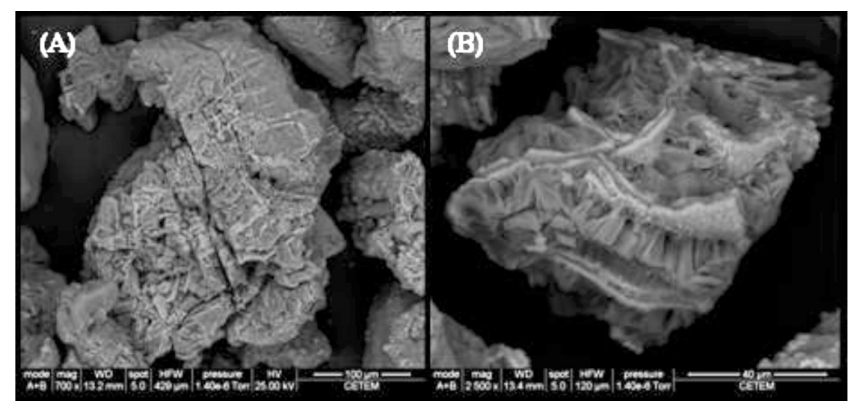

Figura 9: Micrografias obtidas por microscopia eletrônica de varredura da fração BFG2: (A) evidências de rocha alterada e (B) montmorillonita com veios de hematita.

[Figure 9: SEM micrographs of the BFG2 fraction: (A) signals of modified rock and $(B)$ montmorillonite with veinlets of hematite.]

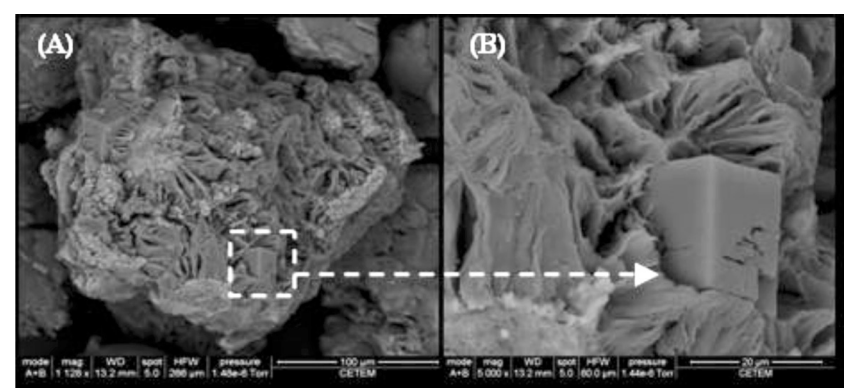

Figura 10: Micrografias obtidas por microscopia eletrônica de varredura da fração BFI: (A) aglomerado e (B) destaque da morfologia prismática típica de K-feldspato.

[Figure 10: SEM micrographs of the BFI fraction:(A) agglomerated and (B) typical prismatic morphology of a K-feldspar crystal.]

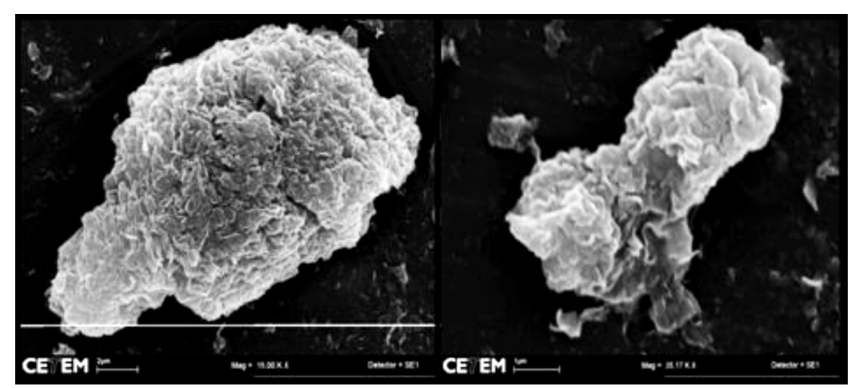

Figura 11: Micrografias obtidas por microscopia eletrônica de varredura da fração BFF2: aglomerados com lamelas típicas de montmorillonita.

[Figure 11: SEM micrographs of the BFF2 fraction: agglomerates with typical lamellae of montmorillonite.]

difratogramas da BFI e BFMAG; - o teor elevado de $\mathrm{Fe}_{2} \mathrm{O}_{3}$ nas frações BFG1 e BFG2 está relacionado à presença de magnetita e principalmente hematita. A magnetita só é detectada no difratograma da BFMAG, pois está em baixíssima concentração.

Com as análises de MEV (Figs. 8, 9, 10 e 11), é possível entender o porquê da montmorillonita estar presente em todas as frações granulométricas, mesmo na fração mais grossa (BFG1). As micrografias revelaram detalhes texturais surpreendentes dos minerais formadores da bentonita Formosa. Na Fig. 8 observa-se nitidamente a presença de rosetas de montmorillonita envolvidas por hematita em hábito tipicamente botrioidal. Na Fig. 9 temse o retrato típico de uma rocha alterada quimicamente, com fissuras de dissolução preenchidas por botrióides de hematita e matriz feldspática tranformada em montmorillonita. Na Fig. 10 observa-se, além das lamelas montmorilloniticas e botrioidais de hematita, a morfologia prismática de um cristal de K-feldspato. Nas micrografias da fração argila (BFF2), Fig. 11, observa-se, mesmo com

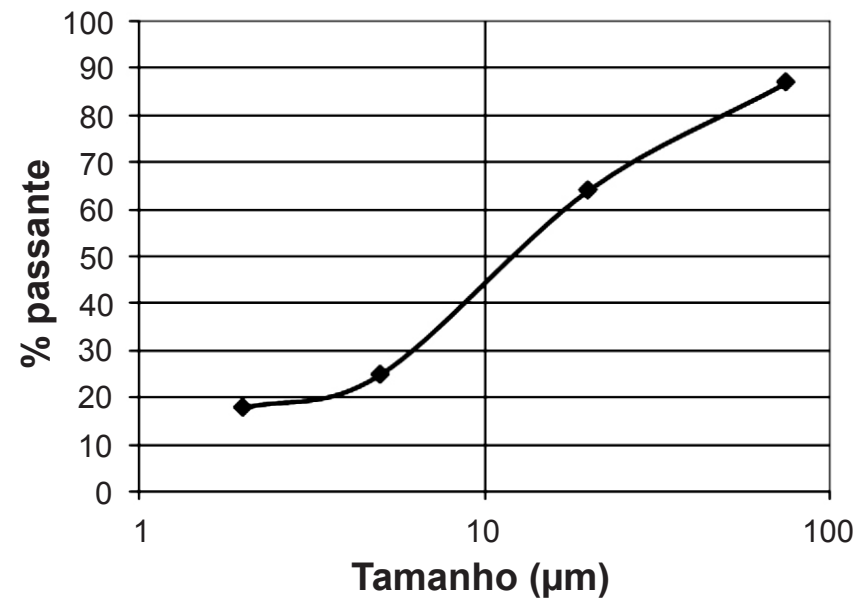

Figura 12: Distribuição de tamanho de partícula da bentonita Formosa.

[Figure 12: Particle size distribution of Formosa bentonite.]

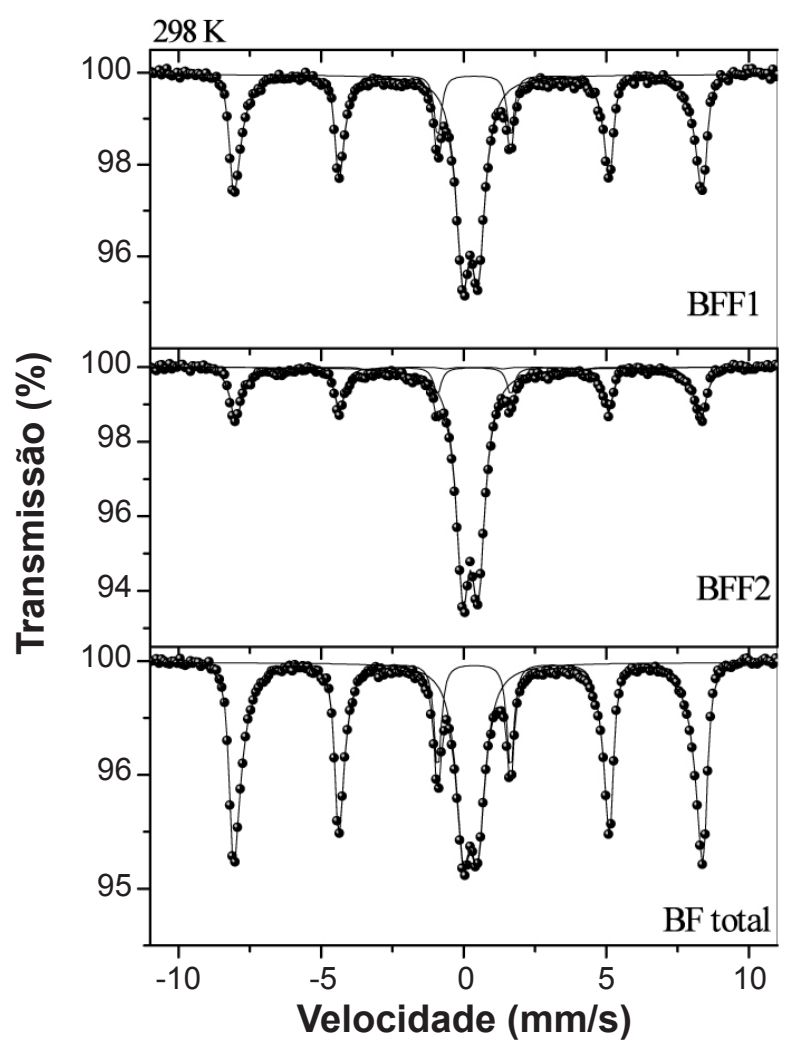

Figura 13: Espectros Mössbauer das amostras BF (amostra total) e frações BFF1 e BFF2.

[Figure 13: Mössbauer spectra of the BF (bulk sample) and BFF1 and BFF2 fractions.] 
Tabela VI - Parâmetros hiperfinos determinados a $298 \mathrm{~K}$. [Table VI - Hyperfine Parameters determined at 298 K.]

\begin{tabular}{ccccccc}
\hline Frações & $\begin{array}{c}\mathrm{H}_{\mathrm{hf}} \\
(\mathrm{kOe})\end{array}$ & $\begin{array}{c}\text { Área } \\
(\%)\end{array}$ & $\begin{array}{c}\mathrm{H}_{\mathrm{hf}} \\
(\mathrm{kOe})\end{array}$ & $\begin{array}{c}\text { Área } \\
(\%)\end{array}$ & $\begin{array}{c}\Delta \mathrm{E}_{\mathrm{O}} \\
(\mathrm{mm} / \mathrm{s})\end{array}$ & $\begin{array}{c}\text { Área } \\
(\%)\end{array}$ \\
\hline BF & 511 & 66 & - & - & 0,51 & 34 \\
$\mathrm{BFF} 1$ & 511 & 52 & - & - & 0,54 & 48 \\
$\mathrm{BFF} 2$ & 505 & 31 & 400 & 3 & 0,55 & 66 \\
\hline
\end{tabular}

pouca resolução da imagem devido ao tamanho muito pequeno das partículas, lamelas típicas da montmorillonita aglomerada.

A curva da Fig. 12 indica uma distribuição regular dos tamanhos de partículas, entre 75 e $5 \mu \mathrm{m}$, que pelo observado nas imagens, advém da má liberação dos grãos, pelo efeito da cimentação ferruginosa, e, principalmente, pela aglomeração dos argilominerais. Desta forma, apesar de formar cristais muito finos, a montmorillonita domina a assembléia mineralógica até da fração mais grossa analisada.

Os espectros Mössbauer da BF (amostra total) e de suas frações finas (BFF1 e BFF2) são similares e apresentam basicamente as mesmas características: um sexteto relativamente estreito e um dubleto relativamente largo (Fig. 13). Apesar das análises em temperatura ambiente não serem conclusivas, fica claro que o ferro ocorre no mínimo em dois minerais diferentes, hematita e montmorillonita. O sexteto estreito refere-se à hematita, e os valores dos campos magnéticos hiperfinos (Tabela VI), sugerem substituições isomórficas inferiores a $10 \%$, desde que se ignore o efeito de pequeno tamanho de partícula. Além disso, pode-se observar, pelas intensidades dos picos, que hematita está em menor concentração em BFF2 (fração argila), o que é justificado por se tratar de uma amostra mais purificada. O dubleto largo é atribuído a montmorillonita contendo ferro, bem similar ao descrito por Aranha [21] para a bentonita Chocolate.

\section{CONCLUSÕES}

O ponto fundamental na caracterização do basalto intemperizado estudado, visando a sua utilização como bentonita, está na presença dominante de montmorillonita e baixa concentração de outras fases minerais. Em todas as frações estudadas (amostra total e fracionada) ela foi dominante, o que pode atender o uso em qualquer uma das formas, dependendo da exigência da aplicação. A distribuição de massa não realçou a predominância de uma fração granulométrica, porém montmorillonita esteve sempre presente como principal mineral em todas as frações, denotando um elevado estado de aglomeração. Finalmente, a composição química e CTC revelaram tratarse de uma (Mg)-montmorillonita. Pretende-se, em trabalhos futuros determinar às propriedades reológicas da bentonita Formosa, tais como: viscosidade, densidade, capacidade de inchamento, dentre outras. Esses parâmetros definirão sua melhor aplicação, se na indústria petrolífera, siderúrgica, fundição, tintas, vernizes, construção civil, farmacêutica, cosmético e/ou outras. Essas propriedades devem ser determinadas em amostras in natura e em amostras tratadas sodicamente, já que a caracterização mostrou tratar-se de uma (Mg)-montmorillonita. Vale lembrar que as principais bentonitas do Brasil, Chocolate e Bofe, que representam cerca de $80 \%$ da bentonita produzida no país, também não são sódicas, e tem que ser ativadas com carbonato de sódio para atender as exigências do mercado, principalmente para as aplicações como fluidos de perfuração e pelotização.

\section{AGRADECIMENTOS}

Ao $\mathrm{CNPq}$ pelo auxilio financeiro: bolsa de mestrado da primeira autora (Proc. 131.752/2008-1) e pela bolsa de produtividade em pesquisa do segundo autor (Proc. 304.566/2007-1), e à CAPES pelo auxilio financeiro (Proc. 10700005).

\section{REFERÊNCIAS}

[1] P. T. Vaz, N. G. A. M. Rezende, J. R. Wanderley filho, W. A. S. Travassos, Boletim de Geociências da Petrobrás, 15 (2007) 253.

[2] A. B. Luz, S. L. M. Almeida, Rochas \& Minerais Industriais, $2^{\mathrm{a}}$ Ed., CETEM/MCT, Rio de Janeiro, RJ (2008) 223.

[3] N. G. A. M. Rezende, Argilas Nobres e Zeólitas na Bacia do Parnaíba, Relatório final, CPRM, Rio de Janeiro, RJ (1997).

[4] D. S. Moraes, R. S. Angélica, C. E. F. Costa, G. N. Rocha Filho, J. R. Zamian, Appl. Clay Sci. 48 (2010) 475.

[5] A. V. Vargas, Alteração supergênica dos basaltos de Porto Franco - Grajaú - MA, Diss. Mestrado, UFPA, Belém, PA (1985).

[6] S. P. A. Paz, Caracterização química, mineralógica e aplicações potenciais da bentonita associada a basaltos intemperizados da Formação Mosquito, bacia do Parnaíba, sul do Maranhão, Diss. Mestrado, UFPA, Belém, PA (2010). [7] P. Souza Santos, Estudo tecnológico de argilas montmoriloniticas do distrito de Boa Vista, município de Campina Grande, Paraíba, Tese de Cátedra, EPUSP, S. Paulo, SP (1968).

[8] J. Elzea, H. H. Murray, Industrial Minerals e Rocks, Society for Minig Metallurgy and Exploration, Littleton, CO, EUA (1994) 125.

[9] C. J. B. Mott, Catalysis Today 2 (1988) 199.

[10] D. M. Moore, R. C. Reynolds Jr., X-ray diffraction and the identification of clay minerals, $2^{\mathrm{a}}$ Ed., Oxford University Press, New York, NY, EUA (1997).

[11] P. Souza Santos, Ciência e Tecnologia de Argilas, vol. 1, 2 ${ }^{\text {a }}$ Ed., Edgar Blücher, S. Paulo, SP (1989).

[12] D. P. Lira Filho, Perfil Analítico da Bentonita, Boletim 04, Departamento Nacional da Produção Mineral, Ministério das Minas e Energia, Rio de Janeiro, RJ (1973).

[13] R. F. Grim, Clay Mineralogy, 2a Ed., McGraw Hill Book Company, New York, NY, EUA (1968). 
[14] R. R. Menezes, P. M. Souto, L. N. L. Santana, G. A. Neves, R. H. G. A. Kiminami, H. C. Ferreira, Cerâmica 55 (2009) 163.

[15] E. Teixeira-neto, A. A. Teixeira-neto, Química Nova 32 (2009) 809.

[16]A.B.Luz,C.H. Oliveira, Rochas \& Minerais Industriais, $2^{\mathrm{a}}$ Ed., CETEM, MCT, Rio de Janeiro, RJ (2008) 239.

[17] M. M. Rezende, Bentonita, In Sumário Mineral 2009, DNPM, Distrito Federal, DF (2009).

[18] P. T. Hang, G. W. Brindley, Clays Clay Minerals 18 (1970) 203.

[19] P. Salerno, S. Mendioroz, Appl. Clay Sci. 22 (2002)
115.

[20] A. Yildiz, M. Kuscu, Clay Minerals 42 (2007) 399.

[21] I. B. Aranha, Preparação, caracterização e propriedades de argilas organofilicas, Tese Dr., UFRJ, Rio de Janeiro, RJ (2007).

[22] L. V. Amorim, C. M. Gomes, H. L. Lira, K. B. França, H. C. Ferreira, Mater. Res. 7 (2004) 583.

[23] M. R. M. P. Aguiar, A. C. Novaes, Química Nova 25 (2002) 1145.

[24] O. Fesharaki, E. García-Romero, J. Cuevas-González, N. López-Martínez, Clay Minerals 42 (2007) 187.

(Rec. 08/09/2010, Ac. 28/01/2011) 